\title{
Attention-deficit/hyperactivity disorder (ADHD): A review of neuroscientific evidence of developmental delay and dysfunction
}

\author{
Jessica J. Stephens* and Dana L. Byrd \\ Department of Psychology and Sociology, Texas A\&M University - Kingsville, USA
}

\begin{abstract}
The childhood onset of the symptom of inattention, often presented along with the symptoms of hyperactivity and/or impulsivity, occurring with severity beyond what is developmentally appropriate, has for decades been a sufficient basis for a psychological or psychiatric diagnosis of Attention Deficit Disorder (ADD) or Attention-Deficit/Hyperactivity Disorder (ADHD). Differences in requirements of symptom severity, symptom combinations, and onset and maturational course of presentation of symptoms has resulted in marked differences in diagnostic prevalence and medical treatment of this group of attention-deficit disorders in children and more recently adults among countries and over the years. The combination of 1) the core aspect of ADD/ADHD being self-control behaviors typical of an individual of younger age, 2) the diagnostic guides allowing for the possibility of change and/or disappearance of some or all symptoms with maturation, and 3) the evolution of the view of these disorders as having valid diagnosis- and treatment-worthy presentation in adulthood, leads to an interesting question, "To what extent is $\mathrm{ADD} / \mathrm{ADHD}$ a developmental delay versus a life-long dysfunction?" This manuscript reviews research findings on the neural basis of attention-deficit disorder or disorders across childhood and into adulthood, with the goal of shedding light on the neurological, maturational, and epigenetic influences over the projection of attention-deficit disorder(s).
\end{abstract}

\section{Background}

When asked to label behavior typical of an average child, common replies might describe impulsiveness, impatience, and heightened energy. These shared mannerisms had by many children could give insight into the present complications and criticisms of accurately identifying and diagnosing Attention-Deficit Hyperactivity Disorder (ADHD). The aforementioned behavioral patterns are characteristic of the affliction, yet it is their strength, duration, associated distress, and dysfunction that may be key in correctly pinpointing the presence of ADHD. Being able to accurately distinguish between children behaving within a normal developmental spectrum and those with a diagnosable ailment may be in need of addressing. Diagnoses in American children remain high, with $12 \%$ of children aged 12-17 being diagnosed currently or formerly with ADHD (NSCH, 2007). Adult prevalence of ADHD (4.4\%) has been reported to be at around half the rate seen in children [1]. This proposes a potential developmental issue at the core of the ADHD phenomenon.

Some questions may remain regarding precisely when and how certain characteristics seen in those diagnosed with ADHD cease to be a part of the normal developmental process. If professionals are diagnosing the young because they show exaggerated childish thinking and behaviors, or childish thoughts and behaviors typical of those at a younger age, what happens when they become mature adults? Do these children outgrow the disorder, along with its associated behaviors and brain functions, or do their atypical behaviors and brain patterns persist, distinctive from those typical of their age group? Does adolescent expression of ADHD change? Are certain environmental factors related to the expression of the disorder and the course it takes in an individual?
In order to better understand the prognosis of ADHD in those afflicted, we must understand the differences in those diagnosed versus those not and the changes in the brain and symptoms over the course of maturation from childhood through adulthood. Neurological testing techniques may provide a window into the disorder, showing the ways in which the brain's structure and activity is different in ADHD and how the behaviors and thinking patterns that are integral to the disorder are likely separate from those of simple immaturity. An overview of the disorder may be requisite for understanding some of these mechanisms, therefore some notes on what a diagnosis of ADHD signifies and how it is identified will follow.

\section{Diagnoses of ADHD in children and adults}

The most recent symptom criteria for ADHD were first described by the American Psychological Association in 2010, when they addressed potential changes to diagnostic parameters for the disorder in the Diagnostic and Statistical Manual of Mental Disorders $5^{\text {th }}$ iteration, which was later published in May of 2013. Symptoms of inattention were defined as conditions within an individual that contribute to trouble with maintaining concentration on regular tasks. Impulsivity was described as engaging in behaviors without appropriate consideration. Hyperactivity was related to trouble with

Correspondence to: Jessica Stephens,; Texas A\&M - Kingsville, Department of Psychology and Sociology, MSC117 Station1, Kingsville, Texas 78363, USA, Tel: (361) 593-2701; E-mail: Jessica.Stephens@tamuk.edu

Key words: attention deficit disorder, attention-deficit/hyperactivity disorder, $A D D, A D H D$, neuroscience, review, development, adult add, adult ADHD

Received: April 23, 2016; Accepted: May 06, 2016; Published: May 11, 2016 
calmly or carefully mobilizing or performing tasks.

The first step in the diagnostic process, in the case of a child, is often initiated when a lay adult reports a child's aberrant behaviors to a health care professional. It is most often a teacher referral to a physician that leads to a diagnosis [2]. Sometimes, however, a child will display abnormal behavior in alignment with ADHD while in a physician's clinic and will therefore be referred by their pediatrician, or the child will be brought into a clinic by a worried parent. In a sample of 3,483,089 diagnosed ADHD children, it was discovered by Zarin and colleagues (1998) that a diagnosis of ADHD is much more likely to be given by a primary physician $(75.4 \%)$ than a psychiatrist $(12.4 \%)$ or other mental health specialist (12.2\%).

What manifestations result in diagnosis? In the United States, behaviors must meet criteria put forth by the current version of the Diagnostic and Statistical Manual of Psychiatric Disorders (DSM), a diagnostic guide for all psychological disorders which is written by a committee of experts under the supervision of the American Psychological Association. The DSM has been updated to a fifth edition, known as the DSM-5, as of May, 2013. ADHD criteria underwent minor changes, but retained much of the specifications that had been set forth by the previous DSM-IV edition. These criteria are elaborated on in the passages that follow.

The DSM-V has listed the same basic symptomatic behaviors and patterns of thought required for an Attention Deficit/Hyperactivity Disorder diagnosis as in the prior edition [3]. These include; symptoms being present in at least two environments that the child is commonly exposed to, proof of a disturbance in the child's ability to maintain a routine quality of life or to complete tasks that are necessary for their optimal growth and functioning, as well as presenting symptoms proving not to reside within the make-up of a different diagnosis that would be more urgent or appropriate to treat.

Patients must display at least six criteria from one of two columns, these being either column 1- inattention, or column 2- heightened activity/impulsive behavior, respectively. The symptom criteria of the inattention column include: (1) proneness for making careless errors on and lack of attentiveness to assignments, (2) inability to prolong attention for leisure and educational situations, (3) wandering attention in verbal exchanges, (4) initiating various activities and abruptly abandoning them when interest wanes, ultimately failing to complete them, (5) untidiness, inability to maintain neatness of spaces and/or projects, in combination with time management errors, (6) preference for activities that are brief or simple and averse to complex and time consuming efforts, (7) losing track of important or necessary objects frequently, such as homework, school utensils, cellular phones, money, and the like, (8) abrupt diverting of concentration due to external sensory data or intrusive internal thoughts, (9) regular forgetfulness of tasks that are in need of attention, such as doing assignments, completing housework, attending important events.

Symptom criteria for the energetic/impulsive behavior include: inability to sit still, (2) abruptly leaving environments where sitting and maintaining attention is required, (3) spontaneously sprinting and/or ascending objects in inappropriate settings, (4) yelling or otherwise being disruptively loud during quiet activities, (5) fidgeting, abrupt intrusion in conversation, inability to maintain stillness for extended periods, and impatience, often appearing "wound-up" and "on the move", (6) superfluous and persistent chatter, (7) consistent and intrusive interrupting of others (8) irritation at being delayed or inability to wait for others who will go before them in a queue,
(9) intruding on other people's personal space or privacy, lacking boundaries with other people and their property.

The most striking alteration to the diagnosis of ADHD made in the DSM-V may be in the area of sub-categories for the disorder. Though similar symptoms must be present as with prior editions, subtypes of ADHD have been entirely reformed. The term "ADD", Attention Deficit Disorder without hyperactivity, will no longer be used by physicians to assess a child who does not meet criteria for heightened activity levels and impulsive behaviors. Rather, there are now specific emphases that can define the predominant symptoms present in an ADHD child. Those meeting up to 2 items, but no greater, in column 2 and who meet at least six items in column 1 will be diagnosed as "Predominantly Inattentive Presentation", while those who display at least six symptoms of column 2 without the presence of at least six symptoms of column 1 for over six months will be treated as "Predominantly Hyperactive/ Impulsive". Those with six or more shared symptoms present from both columns will be diagnosed as "Combined Presentation".

While the symptoms of heightened activity and inattention remain unchanged, the DSM-5 has altered the minimum age at which symptoms must have been present. Rather than symptom presence being necessary before the age of 7 , the requirement is now for symptoms to preside before the age of 12 .

While the DSM-5 is utilized in many countries as a treatment reference, it is used exclusively in America. Conversely, the International Coding of Diseases (ICD-10) is often used independently of the DSM-V the world over for the comprehensive classification of maladies. The ICD-10-volume 2 (2003) closely aligns its list of symptoms for meeting a diagnosis of ADHD with that of the DSM for a formal diagnosis of what it coins a hyperkinetic disorder [4]. Hyperkinetic disorders are deemed behavioral and emotional conditions which have an onset of symptoms that begin in childhood. The display of symptoms are written as involving excessive behaviors displays and an inability to sustain it for extended stretches of time, some cognitive deficits involving an inability to regulate ones thoughts and accompanying actions. The ICD-10 differs from the DSM in a couple of key ways. The ICD-10 includes symptom criteria that are secondary to the main symptoms outlined in the disorder. These are a lack of interest or popularity with peers and a reduced overall self-valuation. The ICD also requires that all categories of symptoms are present and were experienced before the age of 7 , not 12 , which aligns more closely with the former DSM-IV (1994).

Internationally, there are other dissenting views from North America on the prevalence rate for the disorder and the explanations for symptoms. The diagnosis has been linked to distinctive factors, with North American medicine focusing on genes and epigenetics to explain ADHD and European medicine relating symptoms of hyperkinesis to neurological impairment or damage. This may be related to the prevalence rates in both continents being vastly different. The diagnosis of Hyperkinetic disorder in Europe remains something of a rarity with a $0.6 \%$ prevalence in children aged 6-17.

In contrast, $\mathrm{ADHD}$ in America is currently one the most common psychological disorders among adolescents and children, with current North American diagnosing of ADHD at around $11 \%$ of those under the age of 17 [5]. Not only this, but prevalence rates for the diagnosis of childhood ADHD have varied wildly over the years from 3\%-11\% in $1997,4.2-6.3 \%$ in 2003 , and $5.29 \%$ in 2008 , with a mean score of $5.96 \%$ over the course of eleven years. The Centers for Disease Control and Prevention [5] revealed in their findings from national survey 
compilations that the diagnosis increased in prevalence from $5.5 \%$ in 2003 to $11 \%$ in 2011 . The continuously updated CDC data may highlight diagnostic advancements over time, as well as geographic differences in prevalence rates. The lowest rate of ADHD reported was in the state of Nevada, at $5.6 \%$ of children. The highest rate of ADHD was reported in the state of North Carolina, at $15.6 \%$ of children. These stark differences in prevalence rates between states may account for some of the different figures reported between smaller scale studies isolated to certain geographic locations and larger scale studies encompassing a broader population.

\section{ADHD childhood demographics}

Understanding the demographics associated with those diagnosed with the disorder might provide further understanding of the affliction. ADHD is currently one of the most commonly diagnosed psychological disorders among adolescents and children in America. Dependent upon the study and scale, prevalence rates for the diagnosis of childhood ADHD have varied widely over the years from $3 \%-11 \%$ in $1997,4.2-6.3 \%$ in 2003 , and $5.29 \%$ in 2008 , with a mean score of $5.96 \%$ over the course of eleven years. However, these figures conflict with a recent report by the Centers for Disease Control and Prevention [5] based upon their findings from national survey compilations. It was revealed that the diagnosis increased in prevalence from $5.5 \%$ in 2003 to $9.5 \%$ in 2007. The lowest rate of ADHD reported was in the state of Nevada, at $5.6 \%$ of children. The highest rate of ADHD was reported in the state of North Carolina, at $15.6 \%$ of children. These stark differences in prevalence rates in childhood ADHD diagnoses between states may account for some of the different figures reported between smaller scale studies isolated to certain geographic locations and larger scale studies encompassing a broader population. The demographics information presented by the CDC suggests that income disparity seems to play a limited role in diagnostic rates. Children in households at or below poverty level are reportedly diagnosed at a $10 \%$ rate while those in households at or above the poverty level being diagnosed at rates of $11 \%$ [6]. Another aspect of ADHD that may be in need of better understanding is the higher prevalence rate, and higher number of symptoms reportedly expressed, in males over females from childhood thru adulthood [5].

\section{ADHD adult demographics}

Adult ADHD has been formally addressed in the DSM-5. The diagnosis is often categorized as Adult ADHD after childhood ADHD has been found to persist beyond the age of 17. Adult ADHD may be diagnosed with the presence of five, rather than six, symptoms present in either of the two columns. As a diagnosis, the main criteria difference between adult and childhood ADHD is that adults must have experienced symptoms in childhood at some point before the age of 12 , whether or not they were formerly diagnosed. Considering the possible physiological differences between adults and children, this aspect of diagnosing Adult ADHD may be, to some, relatively questionable.

Many children who display hyperactivity, impulsivity, and a lack of attentiveness often exhibit significant symptom reduction, especially in regards to hyperactivity and impulsivity, by the times they reach adulthood. Kessler et al. [1] reported that, based upon the National Comorbidity Survey Replication, the U.S. adult population is estimated as being diagnosed with ADHD at much lower prevalence rates than native children, but at much higher rates than those seen in adults across nations. This inventory was given to a sample of 9,282 participants in order to assess prevalence rates and co-morbid disorders associated with those suffering from adult ADHD in the United States. It was found that the diagnostic frequency of adult ADHD was at $4.4 \%$ of those sampled. This figure makes up slightly less than half of those individuals estimated as being currently diagnosed with childhood ADHD (9-11\%), and can be partially explained by the fact that, until May of 2013, individuals diagnosed with Adult ADHD must have had symptoms present in childhood, before the age of 7 (now 12). This likely means that any individual presenting symptoms of ADHD in adulthood who did not have these symptoms present beginning in childhood will not be diagnosed with Adult ADHD, as it is, categorically, a developmental disorder. The authors noted that, along with a drop in adult frequency of the disorder, symptoms of the disorder lessened substantially as well. Other studies have found even lower rates of an ADHD clinical diagnosis in adults. In a metaregression analysis on the prevalence of adult ADHD diagnosis done by Simon et al. [7], it was reported that only $2.5 \%$ adults were clinically diagnosed with the disorder.

A remaining commonality between childhood and adult ADHD is the population most affected; Caucasian males. This has been implicated as possibly being due to the fact that white children have more apparent expressions of physical actions than peers from different races, or that an instructor of the same ethnicity as the undiagnosed child feels more comfortable discussing behavioral abnormalities with their parents as opposed to parents from a dissimilar ethnic background [8].

\section{Maturation differences in males and females}

Boys are nearly two and a half times more likely than girls to receive a formal diagnosis, with rates of $13.2 \%$ versus $5.6 \%$, respectively [5]. One explanation might be that males are over diagnosed while females are underdiagnosed. It was found by Bruchmüller et al. [9] that with even with a shared diagnosis of ADHD, a boy would be 2-3 times more likely to receive treatment for the disorder than would a girl, possibly due to the fact that symptoms expressed by boys have been reported to be less problematic and intrusive in educational settings. Still, recent reports have suggested that girls display an identical number of symptoms as boys, on average, when diagnosed with the disorder. It was proposed that common beliefs about a "typical" ADHD child being male might influence differences in the clinical diagnoses and treatment processes of boys and girls [9].

Still, others argue that there are biological differences between the two sexes that influence differences in prevalence and progression of ADHD. Obrien et al. have found evidence that developmental and biological aspects may be key to the gender gap. It is more common for boys to display problems with working memory and motor skills than girls. Male children also commonly have a more pronounced inability to suppress urges in responding and receive more reprimanding for acting out inappropriately, contributing to their more common diagnosis of the predominantly hyperactive or combined subtype of the disorder.

Girls, conversely, tended to display more symptoms associated with atypical planning and organizing of higher order cognitive skills. Female children with ADHD often exhibited more problematic symptoms in areas of goal setting and completion, leading to a more common diagnosis of the Predominantly Inattentive Presentation variety of the disorder.

Girls and boys tend to have very different courses of maturation in brain areas such as the frontal lobes, temporal lobes, parietal lobes, and grey matter of the cerebral cortex. Differences in symptoms were 
said to be related to developmental differences in the prefrontal areas, somatosensory cortex, and parietal lobes, mainly. This may explain why girls and boys who share an ADHD diagnosis have different levels of performance on certain cognitive tasks, namely those involved in forethought and intrusive thought management.

Structural MRI's have shown that some of the aforementioned areas of a boy's brain typically matured at a rate that was 1 to 3 years behind that seen in girls, especially in areas of high order cognitive functioning in the cerebral cortex, which are associated with motor task coordination, organizing sensory information, and perceptual responses to the environment. To further investigate the role of age, maturation, and the brain in Attention Deficit Hyperactivity Disorder, it may be good idea to explore the findings of researchers who have studied the structural and functional nature of the brain for child, adolescent, and adult populations.

\section{Neurology and symptoms of childhood ADHD}

The brain's grey matter consists of neuronal (nerve) cell bodies responsible for perceptual and sensory functions, motor tasks, and activation of recall and communication. White matter in the brain is made up almost entirely of glial cells, which are responsible for nourishment and insulation of cell bodies, and myelinated axonal bodies, responsible for quick and coordinated transmission of neuronal signals. While grey matter serves many important functions in the brain that could play a role in ADHD, such as verbal expression, emotional regulation, and sensory perception, white matter, and its role in contributing to higher order cognitive functions within the brain, tends to be the focus of most Diffusion tensor imaging (DTI) studies on ADHD. Both white and grey matter can be studied extensively utilizing diffusion tensor imaging technology (DTI), which measures the flow of water throughout a tissue, providing a visual image of the neural region, especially its structure.

In a study utilizing DTI technology on children with ADHD, Ashtari et al. [10] found that a reduction of white matter volume was related to diminished ability to prolong one's concentration. A white matter volume reduction or lesion in the cerebellum was also found to relate to inhibited executive functioning in abilities like organizing, verbalizing speech, linguistic memory, and kinesthetic utilization. This led the researchers to propose that the cerebellum may be of much greater value to those trying to understating the underlying functional problems associated with ADHD symptom expression.

Irregular communication between certain regions of the brain may further explain some of the differences found between the ADHD and non-ADHD child, as discussed by Krain and Castellanos [11] in their meta-review of literature on the brain development in ADHD children. Noted differences in the brain of the ADHD child were found to be disruptions between the prefrontal lobe and basal ganglia, which work conjointly to regulate responses to environmental stimuli, and a reduction in overall cerebral volume in areas of the brain such as the corpus callosum (the band of tissue connecting the two brain hemispheres necessary for communication between them) and cerebellum, the area of the brain mainly responsible for motor control. It was reported that both grey and white matter in children with ADHD, were of smaller volumes in several areas, mostly those in the left hemisphere, right posterior areas (perception and memory), and the corpus callosum (communication between hemispheres).

Further, compared to healthy control children, it was found that not only was cerebral volume reduced overall in ADHD children (-3.2\%), but that much of this difference (48\%) was reported as being due to the reduction of volume in the frontal lobes, specifically the prefrontal cortex, an area linked with impulsivity, planning, reasoning, judgment, memory, and higher order cognitive functions, such as executive functioning measures. The hemispheres of the cerebellum, necessary for motor tasks, internal timing, and responsiveness shifts, have also been consistently found to be of smaller volume in ADHD children when measured against controls. In their study, Durston et al. [12] found a substantial volume difference in ADHD versus non-ADHD afflicted children $(-4 \%)$ in cerebral and cerebellar regions combined.

Krain and Castellanos [11] also discovered another group difference seen in the brains of three sets of children: control children without ADHD, children with ADHD who were medicated, and children with ADHD who were not medicated. Prescription stimulant usage was identified as the primary source of influence of volume differences in the cerebrums of these children. Both groups of children with ADHD had less volume than the control children of the same sex and age $(-3.2 \%)$ and this statistically significant difference remained constant throughout adolescence. Further, the group which differed in brain volume from the control group the most substantially was the medicated group [11].

It is possible that the children on stimulant medications had smaller brain volumes than their peers before initiating stimulant use, but this was out of the realm of the study. The brain volume of medicated and non-medicated children was not a part of the initial investigation of the researchers, who proposed to look into broad neurological qualities associated with childhood ADHD. Nonetheless, their findings do suggest that stimulant use during cerebral development in children may relate to hindered brain maturation. This may be of import for the growing population of American children on stimulant medication, reported at 3.5\% in 2008 and up from $2.4 \%$ in 1996, most of which are being treated for ADHD [13].

\section{Neurology and symptoms of adolescent ADHD}

ADHD may be associated with disruption of the neural pathways during development from childhood into adolescence. These neural pathways are said to be the neurons collectively known as "white matter" which connect distanced areas of the brain and CNS to each other for message transference, as opposed to grey matter communication, which is believed to be more local), After a diffusion tensor imaging (DTI) investigation of this notion, Konrad and Eickhoff [14] found that the normal developing brain follows a pattern of maturation in a healthy child that is disturbed in a child with ADHD. This design involves maturation of the brain's default mode network (DMN) which is partly related to spontaneous and abstract thoughts becoming more integrated and interconnected as a child matures into an adolescent and then an adult. They propose that this may be one area where the ADHD brain differs. The neurological connections in these pathways may be more highly fragmented and segregated

In a healthy child, the developing brain follows a pattern of maturation that is believed to involve a reduction in connectivity between short-term neural network pathways, or those involved in processing sensory information of the present moment [14]. The pruning of these pathways may, effectively, decrease the capacity of input in the immediate memory. Short-term neural pathways are typically minimized as a child develops into an adolescent so that longterm pathways may more fully mature and replace them. Long-term pathways are believed to allow for stronger and more efficient relay of information stored in permanent memory. These stored memories 
may then be associated with immediate memories. Associating immediate knowledge of the world around us with memories stored all over the brain helps us to solve problems in the present. This process is sometimes referred to broadly as working memory.

To uncover possible associations between ADHD and neural pathway connectivity, Konrad and Eickhoff [14] investigated findings from 18 structural and functional brain studies on participants with and without $\mathrm{ADHD}$. It was determined that short-term neural pathway reduction may be underutilized in the ADHD brain during maturation into adolescence. This reduced pruning of short-term pathways may relate to current sensory information input overwhelming conscious processing, leading to difficulties in sustaining attention and solving complex problems.

Due to limits on the capacity of functional and structural studies on brain maturation, little can be conclusively said about how these connections form and communicate. Still, it was found that, after maturation, several factors in adolescents with ADHD were often atypical from healthy controls [14]. These included hormonal influences on the brain at puberty, myelination (white matter development), connection distances of neural pathways, and connection strength and number in various areas of the brain involved concentration and working memory, such as the fronto-cerebellar region. These findings may need to be further explored in future research on adolescent ADHD before anything definitive can be said about their involvement.

Durston et al. [12] reviewed the literature summarizing developmental changes found consistently in structural MRI studies. It appears that, though the size of the brain does not increase after age 5, there are changes in the proportion of white and grey matter throughout childhood and into adolescence. Myelination of the axons, responsible for speed and efficiency of neuronal message transmission, in the white matter of the frontal and parietal areas increases substantially between the ages of 5 and 17 years. A lack of myelination is attributed to many neurological diseases, such as multiple sclerosis. Symptoms of demyelination include cognitive disruptions and difficulty with control of muscular movements that may parallel symptoms of ADHD.

Researchers $[15,16]$ have, by now, understood that ADHD is a disorder that impair response inhibition, or the ability to stop one's self from performing an action, This symptom of ADHD has most often been assessed using behavioral task monitoring, or "stop-task monitoring", which measures speed of participant reaction and errors accumulated on an activity which primes users to make quick motor decisions. Using this task monitoring test, it was proposed that the brains of adolescents with ADHD would show physical indicators of the disorder in some areas of the brain. Hart et al. [15] discovered that fMRI mapping of patterns in the brain that can indicate differences not present in those without the disorder. A 77\% diagnostic accuracy was said to be reached, with a $90 \%$ accuracy for the 30 ADHD diagnosed adolescents and $63 \%$ accuracy for the 30 control adolescents utilized. The areas of the brain with different activity under an fMRI during stop-task responding were the prefrontal, striatal, and temporo-parietal regions, which were theorized as being developmentally delayed by up to 5 years. A subsequent study which utilized the same brain mapping and behavioral monitoring methods found similar results [16]. Low activation of the frontal and temporo-parietal regions during stop-task monitoring indicated physical differences in the brains of adolescents with ADHD that were not present in healthy controls and were intermediately present in unaffected siblings. If perfected, this type of physical examination of the brain may be a more accurate way of observing and diagnosing the disorder than traditional subjective assessment of symptoms.

\section{Neurology and symptoms of adult ADHD}

Adults with ADHD are found to be consistently different than their child counterparts in symptom expression. Symptom observation in adults included much more variation of inattention, as well as less hyperactivity and impulsivity. ADHD Symptoms uncommon to children included irregularly high mobility between jobs and abnormalities in self-care such as higher instances of addiction to illicit substances and nicotine, insomnia, and poor diet. All variants of ADHD symptoms were said to be less severe, more varied, and less obvious in adults than those in ADHD children, specifically impulsive and hyperactive symptoms, with inattentive symptoms showing less decline [1]. While adults who were diagnosed with ADHD retained many of the difficulties in prolonged concentration, they tended to experience less trouble with sitting still, controlling spontaneous behaviors, or being in structured settings for extended periods. It was concluded that successful therapeutic treatment of childhood $\mathrm{ADHD}$ related to an overall reduction of symptoms in adult $\mathrm{ADHD}$ and controlled for development of many co-morbid disorders such as mood and anxiety disorders.

In their review of Neuropsychological aspects of Adult ADHD, Kaplan and Stevens [17] reported that the most obvious physiological differences between adults with ADHD and controls, as measured by functional Magnetic Resonance Imaging (fMRI), were in frontal lobe areas of the brain, notably a reduction in the size of the right prefrontal cortex and the basal ganglia The prefrontal cortex area of the brain is thought to be responsible for many high order cognitive functions associated with planning, inhibition, attentiveness, judgment, and arousal, while the basal ganglia is believed to be most involved in motor coordination and functioning.

Fair et al. [18] proposed that symptom differences of ADHD that were age-dependent could be due to the differences between children and adults in areas of default neural network formation and function. These neural networks are proposed as being involved in attentional tasks associated with working memory. Children at age six had significantly more trouble than adults in maintaining focus and reducing conscious disruptions. This was believed to be related to the fewer overall connections between networks in the brain, mainly in the the fronto-parietal areas (high order cognitive functioning and somatosensory data) and the rest of the brain. Adolescent brains were found to be developed midway through childhood and adulthood in regards to neural network development, with connections becoming strongest and most numerous in adult neural networks [18]. This finding might imply that with adulthood comes an overall greater ability to maintain attention for extended periods due to development of highly functional working memory networks within the brain.

The frontal lobes (executive functioning tasks) and the basal ganglia (voluntary movement and procedural learning) have been found to be associated with ADHD and show marked growth throughout childhood [12]. It is likely that the maturation of these brain regions contributes to shifts in ADHD symptom development from childhood to adulthood. Even though many symptoms, especially those of the hyperactive and impulsive variety, were often reported as declining with age, inattention deficits remained relatively constant. This was even the case in the majority of the $60 \%$ of individuals who were reported as going through a remission of the disorder and had too few symptoms to garner a formal diagnosis [19]. These findings are consistent with 
much of the research in the area of maturation and ADHD, specifically regarding the decline of symptoms of hyperactivity and impulsivity being earlier and more pronounced than those of inattention.

Other studies on neurological aspects of adult ADHD have utilized Positron Emission Tomography (PET) scans to isolate glucosal level disparities between those with ADHD and healthy controls. These studies have found that the frontal regions of the brain in non-medicated adults with ADHD have reduced glucose levels during completion of tasks associated with higher order cognitive functioning [17]. The tasks performed while measures of brain activity were recorded were associated with working memory, response time during task switching, inhibitory response time, and ability to filter interference, all areas of central executive functioning within the brain. Executive functioning has long been a primary area of interest in many studies which focus on symptomology of ADHD. It was found that the most common differences between Adults with ADHD and Adults without ADHD were consistently in measures of response time (Basal ganglia) higher order cognitive tasks associated with planning and organization (prefrontal lobes), and working memory tasks (default neural networks).

\section{Development or dysfunction?}

The Dynamic developmental theory of ADHD proposed by Sagdvolden et al. proposes that ADHD is caused by a lack of appropriate responding to GABA and glutamate because of deficient dopaminergic actions. These inadequate dopamine activations lead to a reduction in the homeostatic regulation by GABA and Glutamate. This could alter responses to stimuli and result in impairments in the reinforcement of basic behaviors such as motivation, attention regulation, impulse control, planning, and excitability. The behavioral variables most closely associated with cortical dopamine abnormalities are said to be sustained attention deficits and improper organization of goals. Striatal dopamine dysfunction is believed to result in the impairments in organizing learned information and real-time memory associations, as well as a lack of regulation of physical activity. The two aspects of dopaminergic activity in the brain proposed as being dysfunctional in this theory, provides a possible explanation of both inattentive and hyperactive symptoms of ADHD.

Physiological dysfunction may explain symptoms of ADHD as well. Some of these deviation in biological activity were said to relate to neural network interaction impairment in the basal ganglia, responsible for motor learning and coordination of movements, the prefrontal cortex, responsible for problem-solving, impulse control, and maintaining focus, and the thalamus, an area of the brain responsible for alertness while also acting as a sort of sensory switchboard, sending environmental data retrieved by the senses to designated locations within the brain. An irregularity here may lead to less efficient message relay between these areas of the brain, as well as psychophysiological defects, such as abnormal central nervous system (CNS) activity in response to environmental stimuli. These numerous areas of the brain, working in concert, may have combined abnormalities in the ADHD individual that relates to their broad range of central executive deficiencies.

Executive Dysfunction theory is supported by many ADHD researchers, including Barkely, Casstellanos and Tannock, and Pennington and Ozonoff, and Schaechar. This theory describes the central characteristics of the disorder as being involved in deficits of behavioral appropriateness, rationality, attentiveness, forethought, and short term memory retention and processing. In their review of theories of ADHD Johnson et al. [20] explain that executive function theory believes that disparities in the functioning of the basal ganglia, thalamus, and parietal cortices within the brain involved in instinctive cognitions like movement, cross-communication within the brain, and information processing are abnormal in those with ADHD. This is said to be linked with irregularities in fronto-striatal and frontoparietal cortices, associated with motor coordination and attentional processing, respectively, and atypical dopaminergic and noradrenergic transmissions in individuals with ADHD that are not present in those without ADHD. Some argue that this theory focuses the majority of its findings on the impulsiveness and inattentiveness seen in ADHD while largely ignoring much of the excessive activity levels seen in the disorder [20]. A possible explanation of how executive functioning deficits could lead to heightened physical activity and fidgeting may be that these are secondary characteristics of the primary deficits in motor coordination, self-control, and arousal to stimuli. The improper processing of regulatory responses could result in irregular motor activity.

Another concept asserted as a possible explanation for ADHD that has been investigated by Johnson et al. [20] is the State Regulation Model. This theory is directly based upon Sander's model of Cognitive Energy, where task efficiency in basic computations, such as remembering, sensory encoding, and reflexive actions are found to be irregular in those with ADHD. This theory concludes that irregular energy distribution, responsible for certain cognitions, leads to task performance disruptions in those with ADHD due to disruptions in basic computational properties of the brain [20]. It is believed that the ADHD individual is prone to difficulties in regulating and organizing effortful cognitions, especially when information is presented to the child in a slow or periodic manner and a response is required. This is most specifically referred to as problems in activation, or effort needed in order to incline an individual to act. This has been hypothesized as being due to problems in allocating effortful cognitive resources to stimuli in the environment, causing issues of evaluation of environmental events and inhibited response times. Johnson and associates also found that studies could reverse these effortful deficits by having a reward stimulus as incentive for ADHD individuals, as they showed comparable measures to controls without ADHD who also received an incentive. This could indicate that the effortful attentional processes of the ADHD brain may be in need of more meaningful or motivational stimuli in order to act in a comparable way as those in individuals without the disorder. It is also possible that a reduced arousal of cortisol, a steroid associated with stress responses, and not a problem of energy distribution and activation levels within the brain, leads to inhibited activation of certain cognitive responses meant to act on external stimuli [20].

\section{Conclusions}

It was found that separate studies varied drastically on the ongoing clinical diagnosis of ADHD from childhood into adulthood, with ranges of $4-66 \%$ retention rates [7]. This was thought to be due to methodological differences between studies. This was proposed as being due to the studies not utilizing the same diagnostic instruments. Some studies utilized quantitative assessments while others utilized qualitative assessments of symptoms. These methods included symptom check-lists, self-reports, peer-reports, or professional reports used by caregivers and care providers of the child. These may have led to disparities in measurements of inattention, hyperactivity, and impulsivity which could depend upon the diagnostic instruments and individuals administering them for an assessment of symptoms. 
Results from comparison studies of those with and without Adult ADHD can be rife with differences in symptoms type, onset, quantity, and severity, and therefore may be unreliable [1]. Future studies may want to control for this by isolating the diagnostic tools and professionals utilizing these tools when comparing individuals who have been formally diagnosed with ADHD. An additional way to minimize the differences in findings between studies that utilize qualitative self-reports of ADHD symptoms may be to measure quantitative neurological aspects of the disorder in individuals.

\section{References}

1. Kessler RC, Adler L, Barkley R, Biederman J, Conners CK, et al. (2006) The prevalence and correlates of adult ADHD in the United States: results from the National Comorbidity Survey Replication. Am J Psychiatry 163: 716. [Crossref]

2. Ghanizadeh A (2009) Psychiatric comorbidity differences in clinic-referred children and adolescents with ADHD according to the subtypes and gender. $J$ Child Neurol 24: 679-684. [Crossref]

3. American Psychiatric Association (2013) Diagnostic and Statistical Manual of Menta Disorders (DSM-5®). American Psychiatric Pub.

4. World Health Organization (2016) ICD Version: 2016. International Statistical Classification of Diseases and Related Health Problems 10th Revision.

5. Centers for Disease Control and Prevention (2011) Attention Deficit/Hyperactivity Disorder. Data \& Statistics.

6. Akinbami LJ, Liu X, Pastor PN, Reuben CA (2011) Attention deficit hyperactivity disorder among children aged 5-17 years in the United States. NCHS Data Brief 70: 1-8. [Crossref]

7. Simon V, Czobor P, Bálint S, Mészáros A, Bitter I (2009) Prevalence and correlates of adult attention-deficit hyperactivity disorder: meta-analysis. $\operatorname{Br} J$ Psychiatry 194: 204-211. [Crossref]

8. Reid R, Riccio CA, Kessler RH, Dupaul GJ, Power TJ, et al. (2000) Gender and ethnic differences in ADHD as assessed by behavior ratings. J Emoti Behav Disord 8: 38-48.

9. Bruchmüller K, Margraf J, Schneider S (2012) Is ADHD diagnosed in accord with diagnostic criteria? Overdiagnosis and influence of client gender on diagnosis. $J$ Consult Clin Psychol 80: 128. [Crossref]

10. Ashtari M, Kumra S, Bhaskar SL, Clarke T, Thaden E (2005) Attention-deficit/ hyperactivity disorder: a preliminary diffusion tensor imaging study. Biol Psychiatry 57: 448-455. [Crossref]

11. Krain AL, Castellanos FX (2006) Brain development and ADHD. Clin Psycho Review 26: 433-444.

12. Durston S, Hulshoff Pol HE, Casey BJ, Giedd JN, Buitelaar JK, et al. (2001) Anatomical MRI of the developing human brain: what have we learned. $J$ Am Acad Child Adolesc Psychiatry 40: 1012-1020. [Crossref]

13. Zuvekas SH, Vitiello B (2012) Stimulant medication use in children: a 12-year perspective. Am J Psychiatry 169: 160-166. [Crossref]

14. Konrad K, Eickhoff SB (2010) Is the ADHD brain wired differently? A review on structural and functional connectivity in attention deficit hyperactivity disorder. Hum Brain Mapp 31: 904-916. [Crossref]

15. Hart H, Chantiluke K, Cubillo AI, Smith AB, Simmons A (2014) Pattern classification of response inhibition in ADHD: toward the development of neurobiological markers for ADHD. Hum Brain Mapp 35: 3083-3094. [Crossref]

16. van Rooij D, Hoekstra PJ, Mennes M, von Rhein D, Thissen AJ, et al. (2015) Distinguishing Adolescents With ADHD From Their Unaffected Siblings and Healthy Comparison Subjects by Neural Activation Patterns During Response Inhibition. Am J Psychiatry . [Crossref]

17. Kaplan RF, Stevens M (2002) A review of adult ADHD: a neuropsychological and neuroimaging perspective. CNS Spectr 7: 355-370. [Crossref]

18. Fair DA, Cohen AL, Dosenbach NU, Church JA, Miezin FM, et al. (2008) The maturing architecture of the brain's default network. Proc Natl Acad Sci U S A 105 : 4028-4032. [Crossref]

19. Biederman J, Mick E, Faraone SV (2000) Age-dependent decline of symptoms of attention deficit hyperactivity disorder: impact of remission definition and symptom type. Am J Psychiatry 157: 816-818. [Crossref]

20. Johnson KA, Wiersema JR, Kuntsi J (2009) What would Karl Popper say? Are curren psychological theories of ADHD falsifiable? Behav Brain Funct 5: 1088-1097. [Crossref]

Copyright: (C2016 Stephens JJ. This is an open-access article distributed under the terms of the Creative Commons Attribution License, which permits unrestricted use, distribution, and reproduction in any medium, provided the original author and source are credited. 